\title{
La salud dental de los niños y adolescentes de Navarra, 2007 (4 ${ }^{\mathrm{a}}$ edición)*
}

\author{
Dental health of children and adolescents of Navarra, 2007 \\ (4 ${ }^{\text {th }}$ edition)
}

\author{
F.J. Cortés, J. Artázcoz, E. Rosel, P. González, M.A. Asenjo, I. Sáinz de Murieta, M. Bravo
}

\section{RESUMEN}

Fundamento. Transcurridos 5 años desde la última encuesta (2002), se procede a una nueva edición que supone la $4^{\mathrm{a}}$ desde 1987. El objetivo es conocer el estado de salud oral de niños y adolescentes de Navarra y su evolución.

Población y métodos. La muestra ( $\mathrm{n}=1.397)$ fue seleccionada mediante muestreo probabilístico polietápico, tomando como unidad las aulas de $1^{\circ}$ y $6^{\circ}$ de Enseñanza Primaria y de $2^{\circ}$ de Enseñanza Secundaria Obligatoria. El trabajo de campo se realizó entre los meses de abril y mayo de 2007 por dos equipos examinadores calibrados.

Variables de salud estudiadas: caries (medida con criterio OMS y con criterio ampliado), estado periodontal y maloclusión. Los datos fueron informatizados con Access XP 2003 SP3; el análisis estadístico, con SPSS Windows v.15.0.

Resultados. El 10,8\% de la muestra es de origen extranjero. La prevalencia de caries en dentición temporal a los 6 años es $35,9 \%$ con cod 1,26; en dentición permanente, $28,5 \%$ y $46,4 \%$ a los 12 y 14 años; la media de dientes afectados 0,63 y 1,28 respectivamente. El índice de restauración es $26,1 \%$ en dentición temporal y 68,9 y $74,1 \%$ en permanente; los de origen extranjero presentan más caries $(p<0,05)$ y menos tratamiento $(\mathrm{p}<0,05)$ que los de origen español. El $62,8 \%$ de 14 años presentan sangrado gingival y el 16,8\%, cálculo; el $14,7 \%$ maloclusión y el $19,1 \%$ lleva ortodoncia.

Conclusiones. La caries está estabilizada en dentición temporal en población general, pero desciende significativamente entre los autóctonos (origen España); en dentición permanente continúa con su tendencia descendente, aunque más moderada. Navarra está entre las regiones con menor nivel de caries y más alto índice de restauración. Los inmigrantes presentan significativamente más caries. El índice de restauración desciende ligeramente respecto a años anteriores. Las cifras de salud periodontal son pobres. Maloclusión y portadores de ortodoncia están estabilizados.

Palabras clave. Escolares. Salud oral. Caries dental. Tratamiento. Estado periodontal.

\begin{abstract}
Background. With five years having passed since the last survey (2002), a new survey was carried out, the $4^{\text {th }}$ since 1987. The aim is to determine the state of oral health of children and adolescents in Navarre and its evolution.
\end{abstract}

Population and methods. The sample $(\mathrm{n}=1.397)$ was selected through multistage probability sampling, taking as a unit the classrooms of the $1^{\text {st }}$ and $6^{\text {th }}$ year of Primary Education and the $2^{\text {nd }}$ of Compulsory Secondary Education. The fieldwork was carried out in the months of April and May 2007 by two calibrated teams of examiners.

Health variables studied: caries (measured with WHO criterion and extended criterion), periodontal state and malocclusion. The data were computerised with Access XP 2003 SP3; the statistical analysis with SPSS Windows v.15.0.

Results. Ten point eight percent of the sample is of foreign origin. The prevalence of caries in temporary dentition at 6 years is $35.9 \%$ with a dft of 1.26 ; in permanent dentition, $28.5 \%$ and $46.4 \%$ at 12 and 14 years; the average of affected teeth 0.63 and 1.28 respectively. The index of restoration is $26.1 \%$ in temporary dentition and 68.9 and $74.1 \%$ in permanent; those of foreign origin show more caries $(\mathrm{p}<0.05)$ and less treatment $(\mathrm{p}<0.05)$ than those of Spanish origin. Sixty-two point eight percent of 14 year olds show gingival bleeding and $16.8 \%$, calculus; $14.7 \%$ malocclusion and $19.1 \%$ wear orthodontic appliances.

Conclusions. Caries are stabilised in temporary dentition in the general population, but fall significantly amongst natives (those of Spanish origin); in permanent dentition they continue their tendency to fall, although more moderately. Navarre is amongst the regions with a lower level of caries and a higher index of restoration. Immigrants show significantly more caries. The index of restoration falls slightly with respect to previous years. The periodontal health figures are poor. Malocclusion and orthodontic appliance wearers are stabilised.

Key words. Schoolchildren. Oral health. Dental caries. Treatment. Periodontal status.

\section{An. Sist. Sanit. Navar. 2009; 32 (2): 199-215}

* Proyecto financiado por el Departamento de Salud del Gobierno de Navarra y galardonado con la Beca Mikel Larumbe Zazu 2006 (Resolución 2131/2006, de 7 de noviembre, del Director General de Salud).

Recepción: 25 de febrero de 2009

Aceptación provisional: 27 de abril de 2009

Aceptación definitiva: 8 de junio de 2009

\author{
Correspondencia \\ F. Javier Cortés Martinicorena \\ Navas de Tolosa, 27-1 ${ }^{\circ}$ \\ 31002 Pamplona. España \\ Tfno. +34948225725 \\ E-mail: icortes@infomed.es
}




\section{INTRODUCCIÓN}

Durante el año 2007 se llevó a cabo la $4^{\text {a }}$ encuesta epidemiológica de salud oral de la población infantil de Navarra; las anteriores datan de los años $1987^{1}, 1997^{2,3}$ y $2002^{4}$. Han transcurrido, por tanto, 20 años desde la primera toma de datos realizada con metodología estandarizada y una misma dirección de proyecto. Esta serie continuada tiene la capacidad de mostrar con rigor la evolución de las enfermedades dentales más comunes y su tratamiento entre la población infantil y adolescente, así como la de los hábitos relacionados con la salud oral.

Las dos primeras encuestas se centraron en mostrar cuál era el estado de salud dental de esta población y sus necesidades de tratamiento. Posteriormente, la encuesta del año 2002 evaluó el impacto del Programa de Asistencia Dental Infantil (PADI) ${ }^{5}$. En esa encuesta se hizo patente por primera vez en nuestra Comunidad, el fenómeno de la inmigración contabilizando en la muestra un 5,5\% de escolares de origen extranjero. En las conclusiones de aquel estudio se destacaba que la caries seguía su tendencia descendente o estabilizada según grupos, se atenuaban las diferencias anteriormente existentes según nivel socio económico y medio urbano/rural, el nivel de tratamiento aumentaba por efecto del PADI, mejoraban los hábitos higiénicos aunque todavía lejos de un ideal y mejoraba la utilización del PADI en todos los grupos de edad y condiciones sociodemográficas. Un dato destacable fue que los escolares de origen extranjero mostraban unos niveles de caries entre 1,4 y 4,7 veces más que sus homólogos de origen autóctono.

El PADI lleva en funcionamiento desde el año 1991 y cubre a todos los residentes en Navarra entre las edades de 6 y 18 años. En 2007 tuvo un índice de utilización global del $66,7 \%$ lo que supuso atender a un total de 48.388 niños y jóvenes del total de 72.500 con derecho a prestación, de los cuales, el $4,6 \%$ lo fue a través de la red pública y el $95,4 \%$ de la red privada. La provisión se realiza actualmente a través de 3 centros de la red pública (5 dentistas repartidos entre Pamplona, Tudela y Estella) y 181 de la red privada concertada ${ }^{6}$ (que emplean a 197 dentistas).

El objetivo del presente trabajo es conocer el estado de salud oral de los niños y adolescentes de Navarra y su evolución desde la $1^{\underline{a}}$ encuesta.

\section{POBLACIÓN Y MÉTODOS}

La investigación ha sido conducida desde la Fundación Miguel Servet y el Servicio Navarro de Salud/Osasunbidea.

La población objeto de estudio ha estado constituida por todos los niños escolarizados de Navarra que cursan estudios de $1^{\circ}$ a $6^{\circ}$ de Enseñanza Primaria (EP) y $2^{\circ}$ de Enseñanza Secundaria Obligatoria (ESO). En esta edición, y debido a los cambios socio-demográficos acaecidos en la última década, se ha considerado necesario renovar la muestra de colegios que se arrastraba desde la selección de 1987. Para ello, a partir del listado completo de centros educativos del curso académico 2006-07, proporcionado por la Consejería de Educación del Gobierno de Navarra ( $n=235$ centros de educación primaria y $\mathrm{n}=91$ de educación secundaria), se realizó un muestreo probabilístico polietápico con las siguientes características:

- Se definieron 4 estratos (centro público/privado y capital/provincia).

- Se seleccionaron los centros escolares proporcionalmente al número de aulas por curso.

- Se extrajo una sola aula seleccionada aleatoriamente (si sólo había un aula por curso, se selecciona directamente) por colegio y curso y todos los escolares de esa aula se seleccionan para el estudio. De esta forma se obtiene una muestra con equiprobabilidad de selección del escolar.

La selección fue al azar para los centros de primaria (EP) y los de secundaria (ESO) fueron seleccionados según mayor proximidad a aquéllos (cuando fue posible, en el mismo pueblo o centro), para facilitar la logística del proyecto. En escolares de EP 
$\left(1^{\circ}\right.$ y $\left.6^{\circ}\right)$ se calculó para conseguir un tamaño efectivo final de unos 400 escolares (tras considerar una tasa de respuesta del 85\%). En escolares de ESO ( $\left.2^{\circ}\right)$ se calculó para conseguir un tamaño efectivo final de 400 escolares (tras considerar una tasa de respuesta del 95\%). El muestreo se basó en un mínimo de 20 unidades muestrales independientes (clusters o colegios), que es conservador respecto del mínimo de 15 unidades primarias en caso de muestreo por conglomerados. Se seleccionaron además centros reserva para los casos de negativa del centro a participar. Finalmente, fueron 28 los centros de primaria y 20 los de secundaria que participaron en el proyecto.

\section{Variables a estudio}

- Socio-demográficas: edad; sexo; residencia: urbano/rural (han sido considerados como urbanos: Pamplona y su comarca, Estella, Tafalla y Tudela; como rurales, todos los demás).

- Nivel socioeconómico (NSE), siguiendo la misma metodología de encuestas anteriores, se recogió a través de los cuestionarios de preguntas (a los padres en $1^{\circ}$ y $6^{\circ}$ EP y a los propios escolares en $2^{\circ}$ ESO) mediante la profesión del cabeza de familia, posteriormente asignada a categorías profesionales ${ }^{7} \mathrm{y}$ finalmente clasificado en nivel alto/medio/ bajo (resultado de colapsar de las cinco categoría originales las de Alto y Medio alto en "Alto", conservando la categoría "Medio" y colapsando las de Medio bajo y Bajo en "Bajo").

- Nacionalidad, según el criterio utilizado por el Instituto Nacional de Estadística ${ }^{8}$ en la Encuesta Nacional de Salud (ENS); se recogieron 8 posibles orígenes: 0 España; 1 Unión Europea; 2 Otro país de Europa; 3 Canadá o USA; 4 Otro país de América; 5 Asia; 6 África; 7 Oceanía.

\section{Variables de salud bucodental}

- Prevalencia de caries

- Tratamiento de caries
- Sellados de fisuras

- Estado periodontal (sólo $2^{\circ}$ ESO)

- Maloclusión (sólo $6^{\circ}$ EP y $2^{\circ}$ ESO)

- Ortodoncia: lleva o no lleva tratamiento ortodóncico

- Piercing: lleva o no lleva piercing labial y/o lingual

\section{Recogida de datos, formularios, trabajo de campo}

La ficha de recogida de datos clínicos ha sido la misma de anteriores ediciones ${ }^{2-4}$ con ligeras modificaciones que no afectan al contenido ni metodología de las variables recogidas.

Las exploraciones fueron llevadas a cabo entre los meses de abril y mayo de 2007 en los propios centros escolares, utilizando equipamiento portátil y fuente de luz artificial de tipo "spot" de $150 \mathrm{w}$, en locales especialmente habilitados para ello por los propios centros. Se preservaron las condiciones de asepsia del instrumental utilizado. Todos los colegios fueron informados previamente y solicitada su colaboración Tan sólo un centro denegó su participación. Igualmente, se contó con la autorización de la Dirección General de Educación.

Se solicitó el consentimiento de los padres en pasivo, mediante carta informativa enviada a sus domicilios, dando por entendido que la no respuesta negativa implicaba la autorización.

\section{Criterios diagnósticos}

Al igual que en las encuestas anteriores, el diagnóstico de caries se hizo en dos niveles: (1) caries en dentina y (2) caries incipiente o supuestamente remineralizada. En concreto, los criterios utilizados y contenidos en el protocolo del estudio fueron los siguientes:

1. Caries en dentina (criterio de la OMS $^{9}$ modificado). Se diagnosticaron como tal las siguientes lesiones: lesiones en hoyos, fisuras o super- 
ficies lisas, con fondo o paredes reblandecidas, que presenten cavidad y evidencien la invasión de la dentina. Lesiones bajo el borde marginal interproximal, de aspecto blanquecino o levemente oscuro, que aun sin poder ser exploradas con la sonda, ofrezcan una evidencia clara de caries activa en dentina.

2. Caries incipiente/remineralizada. Se diagnosticaron como tal las siguientes lesiones: lesiones de mancha blanca o marrón, en superficies lisas, que no presenten evidencia de cavidad. Hoyos y fisuras teñidos, oscuros, que no presenten reblandecimiento del fondo o las paredes. Pequeñas lesiones en dentina que presenten una clara evidencia de remineralización (dentina dura de color marrón oscuro).

Estado periodontal. Evaluado mediante el Índice Periodontal Comunitario $(\mathrm{CPI})^{9}$, que sustituye al antiguo CPITN y que en esta edad (14 años) sólo contempla tres grados: sano, sangrado y cálculo. Maloclusión. Mediante el índice simplificado de la $\mathrm{OMS}^{9}$. Portadores de Ortodoncia: se registró si llevaban o no tratamiento de ortodoncia. Piercing: se registró si llevaban o no, uno o más piercings labial o lingual.

\section{Metodología de los índices}

El índice co/CAO es una media y se construye mediante la suma de los dientes cariados, ausentes y obturados (CAO), dividido por el total de sujetos examinados. Expresado en minúsculas se refiere a dentición temporal; en mayúsculas, a dentición permanente. Si toma como unidad el diente se expresa como CAOD y si toma la superficie, como CAOS. Sumando al CAOD el segundo nivel diagnóstico (caries incipiente/remineralizada) se expresa como CAODi. Por último, el SiC (Significant Caries Inde $x^{10}$ ) expresa la media del tercio último de la distribución que tiene mayor índice CAOD.
El índice de restauración (IR) es la fracción de caries del CAOD tratada conservadoramente (obturación o empaste) y se calcula: O/CAODx100. El índice CPI expresa la valoración de cada uno de los tres grados medidos, en términos de porcentaje de sujetos en cada categoría y media de sextantes afectados. La maloclusión se expresa en porcentaje de sujetos de cada categoría diagnóstica.

\section{Entrenamiento y calibración de examinadores}

Las exploraciones bucales fueron realizadas por los mismos dentistas que en las dos últimas ediciones (MAAM y ISMI) ayudados de su auxiliar de clínica. Estos equipos examinadores han sido calibrados en ocasiones anteriores ${ }^{2,4} \mathrm{y}$ también para encuestas de ámbito nacional, por lo que se estimó que no era necesario proceder a una nueva calibración. Sin embargo, sí recibieron una jornada previa de entrenamiento. Con unas semanas de antelación al comienzo del trabajo de campo, ambos equipos recibieron un ejemplar del protocolo del estudio con su manual de instrucciones. Posteriormente el investigador principal (FJCM) mantuvo una reunión para discutir los aspectos relevantes del estudio y repasar, sobre modelos fotográficos, los criterios diagnósticos, especialmente los niveles de caries.

\section{Análisis estadístico y presentación de resultados}

Los datos fueron informatizados con el programa Access XP 2003 (10.6771.6811) SP3, con un sistema automático de detección de errores. El análisis estadístico se ha realizado con el programa SPSS Windows v.15.0 (SPSS Inc, Chicago, Il), y para los índices de restauración, con el procedimiento RATIO de SUDAAN 7.0 [RTI, RTP, $\mathrm{NC}]$. Se han calculado valores descriptivos (medias y porcentajes), errores estándar, intervalos de confianza y pruebas de significación (test de student, chi cuadrado, prueba exacta de Fisher bilateral, ANOVA, 
Kruskal-Wallis y Mann-Whitney), de acuerdo con el tipo de variable siguiendo recomendaciones estadísticas ${ }^{11}$.

\section{RESULTADOS}

La tasa de respuesta de la muestra inicialmente seleccionada fue del $97,54 \%$. El $2,46 \%$ de rechazo corresponde a uno de los centros que rehusó participar en el estudio, por lo que fue sustituido por otro de reserva.
La tabla 1 describe la muestra por edad y variables socio-demográficas; el 89,2\% es de origen español y el 10,8\% de origen extranjero. El análisis de la submuestra de extranjeros por nivel socio-económico (NSE) revela que el $17,9 \%$ es de nivel alto, $6,6 \%$ medio y $75,5 \%$ de nivel bajo (datos no mostrados). Las medias de edad han sido 6,35, 11,42 y 13,47 . En las cabeceras de las tablas se hacen constar los intervalos de edad 6-7, 11-12 y 13-14, pero en el texto, para abreviar, se mencionan los grupos referencia: 6, 12, 14 .

Tabla 1. Muestra.

\begin{tabular}{|c|c|c|c|c|c|c|c|c|}
\hline & \multicolumn{2}{|c|}{ 6-7 años } & \multicolumn{2}{|c|}{$11-12$ años } & \multicolumn{2}{|c|}{ 13-14 años } & \multicolumn{2}{|c|}{ Total } \\
\hline & $\mathbf{n}$ & $\%$ & $\mathbf{n}$ & $\%$ & $\mathbf{n}$ & $\%$ & $\mathbf{n}$ & $\%$ \\
\hline \multicolumn{9}{|l|}{ Edad } \\
\hline 6 años ( $\left.1^{o} \mathrm{EP}\right)$ & 339 & 65,1 & 0 & - & 0 & - & 339 & 24,3 \\
\hline 7 años ( $\left.1^{\circ} \mathrm{EP}\right)$ & 182 & 34,9 & 0 & - & 0 & - & 182 & 13,0 \\
\hline 11 años ( $\left.6^{o} \mathrm{EP}\right)$ & 0 & - & 286 & 58,6 & 0 & - & 286 & 20,5 \\
\hline 12 años ( $\left.6^{\circ} \mathrm{EP}\right)$ & 0 & - & 202 & 41,4 & 0 & - & 202 & 14,5 \\
\hline 13 años ( $\left.2^{\circ} \mathrm{ESO}\right)$ & 0 & - & 0 & - & 207 & 53,4 & 207 & 14,7 \\
\hline 14 años ( $2^{\circ}$ ESO) & 0 & - & 0 & - & 181 & 46,6 & 181 & 13,0 \\
\hline Edad media $( \pm$ de) & \multicolumn{2}{|c|}{$6,35 \pm 0,48$} & \multicolumn{2}{|c|}{$11,42 \pm 0,49$} & \multicolumn{2}{|c|}{$13,47 \pm 0,50$} & & \\
\hline Hombre & 269 & 51,6 & 250 & 51,2 & 198 & 51,0 & 717 & 51,3 \\
\hline Mujer & 252 & 48,4 & 238 & 48,8 & 190 & 49,0 & 680 & 48,7 \\
\hline Urbano & 385 & 73,9 & 347 & 71,1 & 267 & 68,8 & 999 & 71,5 \\
\hline Rural & 136 & 26,1 & 141 & 28,9 & 121 & 31,2 & 368 & 28,5 \\
\hline Alto & 124 & 24,4 & 141 & 29,0 & 115 & 29,9 & 380 & 27,6 \\
\hline Medio & 119 & 23,4 & 138 & 28,4 & 118 & 30,6 & 375 & 27,2 \\
\hline Bajo & 265 & 52,2 & 207 & 42,6 & 152 & 39,5 & 624 & 45,3 \\
\hline Perdidos & 13 & & 2 & & 3 & & 18 & \\
\hline Origen España & 448 & 86,0 & 449 & 92,0 & 349 & 89,9 & 1.246 & 89,2 \\
\hline Origen extranjero* & 73 & 14,0 & 39 & 8,0 & 39 & 10,1 & 151 & 10,8 \\
\hline Unión Europea & 7 & 9,6 & 6 & 15,4 & 3 & 7,7 & 16 & 10,6 \\
\hline Otro país Europa & 11 & 15,1 & 8 & 20,5 & 4 & 10,3 & 23 & 15,9 \\
\hline Canadá o USA & 0 & - & 1 & 2,6 & 0 & - & 1 & 0,7 \\
\hline Otro país América & 46 & 63,0 & 21 & 53,7 & 30 & 76,9 & 97 & 64,2 \\
\hline Asia & 2 & 2,7 & 2 & 5,1 & 0 & - & 4 & 2,6 \\
\hline África & 7 & 9,6 & 1 & 2,6 & 2 & 5,1 & 10 & 6,6 \\
\hline Total & 521 & & 488 & & 388 & & 1.397 & \\
\hline
\end{tabular}

* Se presentan los porcentajes de individuos de origen extranjero con respecto al total de extranjeros. 


\section{Prevalencia de caries}

La prevalencia de caries en dentición temporal a los 6 años es del 35,9\% y sube al $41,3 \%$ considerando también las lesiones incipientes; la media de dientes afectados en el total de la muestra es 1,26 y, considerando también las lesiones incipientes, 1,51. En dentición permanente, la prevalencia es del $28,5 \%$ y $46,4 \%$ a los 12 y 14 años respectivamente y sube al $36,9 \%$ y $55,2 \%$ si consideramos las lesiones incipientes; la media CAOD es 0,63 y 1,28 a los 12 y 14 años respectivamente y el $\mathrm{CAOD}_{\mathrm{i}} 0,89$ y 1,82 . El índice significativo de caries $\mathrm{SiC} 1,87$ y 3,44. Aproximadamente el $65 \%$ son caries oclusales (Tabla 2).

Tabla 2. Prevalencia de caries y medias (índice CAOD/cod), por edades (IC-95\%).

\begin{tabular}{|c|c|c|c|c|c|c|}
\hline \multirow[b]{2}{*}{$\% \mathrm{CAOD} / \operatorname{cod}$} & \multicolumn{2}{|c|}{ 6-7 (DT) } & \multicolumn{2}{|c|}{ 11-12 (DP) } & \multicolumn{2}{|c|}{ 13-14 (DP) } \\
\hline & 35,9 & $(31,8-40,0)$ & 28,5 & $(24,5-32,5)$ & 46,4 & $(41,4-51,4)$ \\
\hline$\% \mathrm{C} / \mathrm{c}$ & 28,0 & $(24,2-31,9)$ & 11,7 & $(8,8-14,5)$ & 14,9 & $(11,4-18,5)$ \\
\hline$\% \mathrm{~A}$ & & & 0,8 & $(0,0-1,6)$ & 1,0 & $(0,0-2,0)$ \\
\hline$\% \mathrm{O} / \mathrm{o}$ & 17,5 & $(14,2-20,7)$ & 21,5 & $(17,9-25,2)$ & 38,9 & $(34,0-43,8)$ \\
\hline$\% \mathrm{CAOD}_{\mathrm{i}} / \operatorname{cod}_{\mathrm{i}}$ & 41,3 & $(37,0-45,5)$ & 36,9 & $(32,6-41,2)$ & 55,2 & $(50,2-60,1)$ \\
\hline$\% \mathrm{C}_{\mathrm{i}} / \mathrm{c}_{\mathrm{i}}$ & 36,3 & $(32,1-40,4)$ & 24,2 & $(20,4-28,0)$ & 33,0 & $(28,3-37,7)$ \\
\hline$\% \operatorname{cod}+\mathrm{CAOD}$ & 37,8 & $(33,6-42,0)$ & 41,8 & $(37,4-46,2)$ & 46,9 & $(41,9-51,9)$ \\
\hline \multicolumn{7}{|l|}{ Medias } \\
\hline $\mathrm{CAOD} / \operatorname{cod}$ & 1,26 & $(1,06-1,46)$ & 0,63 & $(0,52-0,73)$ & 1,28 & $(1,06-1,50)$ \\
\hline $\mathrm{C} / \mathrm{c}$ & 0,93 & $(0,75-1,11)$ & 0,19 & $(0,13-0,24)$ & 0,31 & $(0,21-0,42)$ \\
\hline A & & & 0,01 & $(0,00-0,02)$ & 0,02 & $(0,00-0,03)$ \\
\hline $\mathrm{O} / \mathrm{o}$ & 0,33 & $(0,25-0,40)$ & 0,43 & $(0,34-0,52)$ & 0,95 & $(0,78-1,11)$ \\
\hline $\mathrm{SiC}$ & & & 1,87 & $(1,65-2,09)$ & 3,44 & $(2,97-3.91)$ \\
\hline $\mathrm{CAOD}_{\mathrm{i}} / \operatorname{cod}_{\mathrm{i}}$ & 1,51 & $(1,30-1,73)$ & 0,89 & $(0,76-1,02)$ & 1,82 & $(1,54-2,10)$ \\
\hline $\mathrm{C}_{\mathrm{i}} / \mathrm{c}_{\mathrm{i}}$ & 1,18 & $(0,99-1,37)$ & 0,45 & $(0,36-0,54)$ & 0,86 & $(0,66-1,06)$ \\
\hline $\mathrm{SiC}_{\mathrm{i}}$ & & & 2,55 & $(2,32-2,78)$ & 4,66 & $(4,08-5,24)$ \\
\hline $\mathrm{CAOS} / \mathrm{cos}$ & 1,89 & $(1,52-2,26)$ & 0,82 & $(0,64-1,01)$ & 1,51 & $(1,21-1,82)$ \\
\hline $\mathrm{CAOS} / \cos _{(\mathrm{b}+1)}$ & 0,24 & $(0,14-0,33)$ & 0,14 & $(0,08-0,20)$ & 0,21 & $(0,14-0,28)$ \\
\hline $\mathrm{CAOS} / \cos _{(\mathrm{m}+\mathrm{d})}$ & 0,98 & $(0,77-1,18)$ & 0,15 & $(0,09-0,21)$ & 0,30 & $(0,17-0,42)$ \\
\hline $\mathrm{CAOS} / \cos _{(\mathrm{o})}$ & 0,68 & $(0,56-0,79)$ & 0,53 & $(0,43-0,64)$ & 1,01 & $(0,83-1,18)$ \\
\hline CAOM & & & 0,52 & $(0,43-0,62)$ & 0,82 & $(0,70-0,94)$ \\
\hline $\mathrm{CAOM}_{\mathrm{i}}$ & & & 0,73 & $(0,62-0,84)$ & 1,03 & $(0,89-1,17)$ \\
\hline
\end{tabular}

DT: dentición temporal.

DP: dentición permanente.

i: incluye caries incipiente o supuestamente remineralizada

SiC: índice significativo de caries (Brathall)

b: bucal; l: lingual; m: mesial; d: distal; o: oclusal

Respecto a la distribución, en dentición temporal, el $14,7 \%$ de los escolares acumula el $70,1 \%$ de los dientes co, y en pemanente a los 12 , el $16,4 \%$ acumula el $80,6 \%$ de dientes CAO (Tabla 3).
Los escolares de origen extranjero (inmigrantes) presentan de forma significativa $(\mathrm{p}<0,05)$ más caries que sus homólogos de origen España (autóctonos), tanto en términos de prevalencia como de medias y en todos los grupos de edad (Tabla 4). 
Tabla 3. Distribución porcentual del índice cod/CAOD, por edades.

\begin{tabular}{cccccccccc}
\hline \multirow{2}{*}{$\mathbf{c o d} /$} & \multicolumn{3}{c}{$\mathbf{6 - 7}$ (DT) } & \multicolumn{4}{c}{$\mathbf{1 1 - 1 2}$ (DP) } & \multicolumn{3}{c}{$\mathbf{1 3 - 1 4}$ (DP) } \\
\cline { 2 - 10 } CAOD & $\mathbf{n}$ & \% & \% acu & $\mathbf{n}$ & \% & \% acu. & n & \% & \% acu. \\
\hline 0 & 334 & 64,1 & 64,1 & 349 & 71,5 & 71,5 & 208 & 53,6 & 53,6 \\
1 & 49 & 9,4 & 73,5 & 59 & 12,1 & 83,6 & 69 & 17,8 & 71,4 \\
2 & 44 & 8,4 & 82,0 & 31 & 6,4 & 90,0 & 40 & 10,3 & 81,7 \\
3 & 18 & 3,5 & 85,4 & 21 & 4,3 & 94,3 & 25 & 6,4 & 88,1 \\
4 & 22 & 4,2 & 89,6 & 22 & 4,5 & 98,8 & 21 & 5,4 & 93,6 \\
5 & 14 & 2,7 & 92,3 & 4 & 0,8 & 99,6 & 11 & 2,8 & 96,4 \\
6 & 13 & 2,5 & 94,8 & 1 & 0,2 & 99,8 & 1 & 0,3 & 96,6 \\
7 & 13 & 2,5 & 97,3 & 1 & 0,2 & 100 & 4 & 1,0 & 97,6 \\
8 & 5 & 1,0 & 98,3 & 0 & - & 100 & 4 & 1,0 & 98,7 \\
$>8$ & 9 & 1,8 & 100 & 0 & - & 100 & 5 & 1,4 & 100 \\
\hline
\end{tabular}

cod/CAOD: media de caries en dentición temporal y permanente, respectivamente

DT: dentición temporal.

DP: dentición permanente

*: acumulado

Tabla 4. Prevalencia de caries y medias (índice CAOD/cod), por edades y según origen (IC-95\%).

\begin{tabular}{|c|c|c|c|c|c|c|c|c|c|c|c|c|}
\hline \multirow[b]{3}{*}{$\% \mathrm{CAOD} / \mathrm{cod}$} & \multicolumn{4}{|c|}{ 6-7 (DT) } & \multicolumn{4}{|c|}{ 11-12 (DP) } & \multicolumn{4}{|c|}{ 13-14 (DP) } \\
\hline & \multicolumn{2}{|c|}{ Origen España } & \multicolumn{2}{|c|}{ Origen extranjero } & \multicolumn{2}{|c|}{ Origen España } & \multicolumn{2}{|c|}{ Origen extranjero } & \multicolumn{2}{|c|}{ Origen España } & \multicolumn{2}{|c|}{ Origen extranjero } \\
\hline & 32,4 & $(28,0-36,7)$ & $57,5^{*}$ & $(45,9-69,1)$ & 26,7 & $(22,6-30,8)$ & $48,7^{*}$ & $(32,3-65,1)$ & 43,80 & $(38,6-49,1)$ & $69,2^{*}$ & $(54,1-84,4)$ \\
\hline$\% \mathrm{C} / \mathrm{c}$ & 23,7 & $(19,7-27,6)$ & $54,8^{*}$ & $(43,1-66,5)$ & 9,8 & $(7,0-12,6)$ & $33,3^{*}$ & $(17,9-48,8)$ & 14,00 & $(10,4-17,7)$ & 23,1 & $(9,2-36,9)$ \\
\hline$\% \mathrm{~A}$ & & & & & 0,4 & $(0,0-1,1)$ & $5,1^{*}$ & $(0,0-12,4)$ & 0,60 & $(0,0-1,4)$ & 5,1 & $(0,0-12,4)$ \\
\hline$\% 0 / 0$ & 16,7 & $(13,3-20,2)$ & 21,9 & $(12,2-31,6)$ & 21,6 & $(17,8-25,4)$ & 20,5 & $(7,3-33,8)$ & 37,00 & $(31,9-42,1)$ & $56,4^{*}$ & $(40,1-72,7)$ \\
\hline \% CAODi /codi & 38,4 & $(33,9-42,9)$ & $58,9^{*}$ & $(47,3-70,5)$ & 35,4 & $(31,0-39,9)$ & $53,8^{*}$ & $(37,5-70,2)$ & 52,70 & $(47,5-58,0)$ & $76,9^{*}$ & $(63,1-90,8)$ \\
\hline$\% \mathrm{Ci} / \mathrm{ci}$ & 33,0 & $(28,7-37,4)$ & $56,4^{*}$ & $(44,5-67,8)$ & 22,7 & $(18,8-26,6)$ & $41,0^{*}$ & $(24,9-57,2)$ & 31,80 & $(26,9-36,7)$ & 43,6 & $(27,3-59,9)$ \\
\hline \multicolumn{13}{|l|}{ Medias } \\
\hline $\mathrm{CAOD} / \operatorname{cod}$ & 0,90 & $(0,74-1,06)$ & $3,47^{*}$ & $(2,57-4,36)$ & 0,58 & $(0,47-0,69)$ & $1,15^{*}$ & $(0,63-1,68)$ & 1,16 & $(0,94-1,39)$ & $2,28 *$ & $(1,37-3,20)$ \\
\hline $\mathrm{C} / \mathrm{c}$ & 0,59 & $(0,46-0,72)$ & $3,01^{*}$ & $(2,15-3,87)$ & 0,15 & $(0,10-0,20)$ & $0,64^{*}$ & $(0,20-1,08)$ & 0,30 & $(0,19-0,41)$ & 0,46 & $(0,13-0,79)$ \\
\hline A & & & & & 0,00 & $(0,00-0,01)$ & $0,05^{*}$ & $(0,00-0,12)$ & 0,01 & $(0,00-0,02)$ & $0,08^{*}$ & $(0,00-0,19)$ \\
\hline $0 / 0$ & 0,31 & $(0,23-0,39)$ & 0,45 & $(0,21-0,69)$ & 0,43 & $(0,34-0,52)$ & 0,46 & $(0,12-0,80)$ & 0,86 & $(0,70-1,02)$ & $1,74^{*}$ & $(0,97-2,52)$ \\
\hline $\mathrm{CAOD}_{\mathrm{i}} / \operatorname{cod}_{\mathrm{i}}$ & 1,14 & $(0,97-1,32)$ & $3,77^{*}$ & $(2,81-4,73)$ & 0,82 & $(0,69-0,95)$ & $1,62^{*}$ & $(0,97-2,26)$ & 1,67 & $(1,38-1,96)$ & $3,15^{*}$ & $(2,07-4,24)$ \\
\hline $\mathrm{C}_{\mathrm{i}} / \mathrm{c}_{\mathrm{i}}$ & 0,83 & $(0,69-0,98)$ & $3,77^{*}$ & $(2,81-4,73)$ & 0,39 & $(0,41-0,47)$ & $1,10^{*}$ & $(0,52-1,69)$ & 0,81 & $(0,60-1,01)$ & 1,33 & $(0,60-2,06)$ \\
\hline $\mathrm{CAOS} / \mathrm{cos}$ & 1,19 & $(0,96-1,42)$ & $6,21^{*}$ & $(4,22-8,19)$ & 0,71 & $(0,56-0,87)$ & $2,05^{*}$ & $(0,60-3,51)$ & 1,36 & $(1,06-1,67)$ & $2,87^{*}$ & $(1,53-4,21)$ \\
\hline $\mathrm{CAOS} / \cos _{(\mathrm{b}+\mathrm{l})}$ & 0,08 & $(0,04-0,11)$ & $1,23^{*}$ & $(0,62-1,85)$ & 0,10 & $(0,06-0,14)$ & $0,64^{*}$ & $(0,07-1,21)$ & 0,19 & $(0,11-0,26)$ & 0,41 & $(0,11-0,71)$ \\
\hline $\mathrm{CAOS} / \cos _{(\mathrm{m}+\mathrm{d})}$ & 0,61 & $(0,47-0,74)$ & $3,23^{*}$ & $(2,13-4,34)$ & 0,12 & $(0,07-0,16)$ & $0,49^{*}$ & $(0,00-1,05)$ & 0,27 & $(0,13-0,40)$ & 0,56 & $(0,11-1,02)$ \\
\hline $\mathrm{CAOS} / \cos _{(0)}$ & 0,50 & $(0,41-0,60)$ & $1,74^{*}$ & $(1,25-2,23)$ & 0,50 & $(0,40-0,60)$ & $0,92^{*}$ & $(0,44-1,41)$ & 0,91 & $(0,74-1,08)$ & $1,90^{*}$ & $(1,11-2,68)$ \\
\hline
\end{tabular}

DT: dentición temporal

DP: dentición permanente

i: incluye caries incipiente o supuestamente remineralizada

b: bucal; l: lingual; m: mesial; d: distal; o: oclusal

*: $\mathrm{p}<0,05$ 
El índice de restauración (IR) de la dentición temporal a los 6 años es el $26,1 \%$; en dentición permanente sube al $68,9 \%$ y $74,1 \%$ a los 12 y 14 años respectivamente. Los escolares de origen extranjero presentan significativamente $(\mathrm{p}<0,05)$ menos tra- tamiento que sus homólogos autóctonos, salvo en el grupo de 14 años, en que se igualan (Tabla 5).

El 40\% (aprox.) presentan algún diente sellado y la media para el total de la muestra es de 1,26 y 1,21 a los 12 y 14 años (Tabla 6).

Tabla 5. Índice de restauración global por edades y según origen (IC-95\%).

\begin{tabular}{cccccc}
\hline \multicolumn{2}{c}{ 6-7 (DT) } & \multicolumn{2}{c}{$\mathbf{1 1 - 1 2}$ (DP) } & \multicolumn{2}{c}{$\mathbf{1 3 - 1 4}$ (DP) } \\
\hline 26,1 & $(20,6-31,6)$ & 68,9 & $(61,5-76,4)$ & 74,1 & $(67,6-80,6)$ \\
\hline Origen España & $\begin{array}{c}\text { Origen } \\
\text { extranjero }\end{array}$ & Origen España & $\begin{array}{c}\text { Origen } \\
\text { extranjero }\end{array}$ & Origen España & $\begin{array}{c}\text { Origen } \\
\text { extranjero }\end{array}$ \\
$34,2(27,1-41,3)$ & $13,0^{*}(6,3-19,7)$ & $73,8(66,7-80,9)$ & $40,0^{*}(16,5-63,5)$ & $73,6(66,2-81,1)$ & $76,4(64,6-88,2)$ \\
\hline
\end{tabular}

DT: dentición temporal

DP: dentición permanente

*: $\mathrm{p}<0,05$

Tabla 6. Distribución y media de dientes permanentes sellados, por edades (IC-95\%).

\begin{tabular}{crcrcrc}
\hline & \multicolumn{2}{c}{$\mathbf{6 - 7}$} & \multicolumn{2}{c}{$\mathbf{1 1 - 1 2}$} & \multicolumn{2}{c}{$\mathbf{1 3 - 1 4}$} \\
\hline 0 & 87,7 & $(84,9-90,5)$ & 60,9 & $(56,5-65,2)$ & 59,8 & $(54,9-64,7)$ \\
1 & 1,9 & $(0,7-3,1)$ & 5,7 & $(3,7-7,8)$ & 10,6 & $(7,5-13,6)$ \\
2 & 3,3 & $(1,7-4,8)$ & 7,4 & $(5,0-9,7)$ & 11,1 & $(7,9-14,2)$ \\
3 & 2,5 & $(1,2-3,8)$ & 6,6 & $(4,4-8,8)$ & 5,4 & $(3,2-7,7)$ \\
+4 & 4,6 & $(2,8-6,4)$ & 19,4 & $(15,9-23,0)$ & 13,7 & $(10,0-17,3)$ \\
\hline Media & 0,34 & $(0,26-0,43)$ & 1,26 & $(1,10-1,43)$ & 1,21 & $(1,01-1,41)$ \\
\hline
\end{tabular}

En el interior de la tabla se dan porcentajes que suman siempre $100 \%$.

El 62,8\% presenta sangrado al sondar la encía y, en términos de media de sextantes afectados, 3,27 están sanos y 2,46 presen- tan sangrado. No hay diferencias estadísticamente significativas por origen (Tabla 7).

Tabla 7. Estado periodontal (índice CPI) global y por origen, a los 13-14 años (IC-95\%).

\begin{tabular}{lcccccc}
\hline & \multicolumn{2}{c}{ Sano } & \multicolumn{2}{c}{ Sangrado } & \multicolumn{2}{c}{ Cálculo } \\
\hline $\begin{array}{l}\text { \% CPI máximo } \\
\begin{array}{l}\text { Media sextantes } \\
\text { afectados }\end{array}\end{array}$ & 20,4 & $(16,3-24,4)$ & 62,8 & $(58,1-67,7)$ & 16,8 & $(13,0-20,5)$ \\
\hline & Origen España & $\begin{array}{c}\text { Origen } \\
\text { extranjero }\end{array}$ & Origen España & $\begin{array}{c}\text { Origen } \\
\text { extranjero }\end{array}$ & Origen España & $\begin{array}{c}\text { Origen } \\
\text { extranjero }\end{array}$ \\
$\begin{array}{l}\text { \% CPI máximo } \\
\begin{array}{l}\text { Media sextantes } \\
\text { afectados }\end{array}\end{array}$ & $20,3(16,1-24,6)$ & $20,5(7,3-33,8)$ & $63,7(58,5-68,7)$ & $56,4(40,1-72,7)$ & $16,0(12,2-19,9)$ & $23,1(9,2-36,9)$ \\
\hline $\begin{array}{l}\text { La variable CPI máximo suma siempre 100\%. Las comparaciones Origen España vs Origen extranjero no son significativas. } \\
\text { 3,28 (3,07-3,49) }\end{array}$ & $3,18(2,52-3,84)$ & $2,46(2,26-2,66)$ & $2,46(1,85-2,07)$ & $0,26(0,19-0,34)$ & $0,36(0,10-0,62)$ \\
\hline
\end{tabular}


Entre el $21,7 \%$ y el $14,7 \%$ presentan una maloclusión moderada o severa y entre el $8,4 \%$ y el $19,1 \%$ llevan actualmente tratamiento de ortodoncia, lo que da un total de entre 30-34\% (aprox.) de anomalías sus- ceptibles de/o en tratamiento ortodóncico (Tabla 8).

En esta muestra, sólo 1 sujeto de 12 años y 5 de 14 años eran portadores de algún piercing labial y/o lingual.

Tabla 8. Maloclusión y portadores de tratamiento de ortodoncia, según edad.

\begin{tabular}{lrlrrrc}
\hline & \multicolumn{3}{c}{$\mathbf{1 1 - 1 2}$} & \multicolumn{3}{c}{$\mathbf{1 3 - 1 4}$} \\
\cline { 2 - 7 } & $\mathbf{n}$ & $\mathbf{\%}$ & $\mathbf{I C - 9 5 \%}$ & $\mathbf{n}$ & \% & IC-95\% \\
\hline Normal & 244 & 50,0 & $(45,5-54,5)$ & 152 & 39,1 & $(34,3-44,1)$ \\
Leve & 97 & 19,9 & $(16,3-23,4)$ & 105 & 27,1 & $(22,6-31,5)$ \\
Moderada / Severa & 106 & 21,7 & $(18,0-25,4)$ & 57 & 14,7 & $(11,2-18,2)$ \\
En tratamiento actual & 41 & 8,4 & $(5,9-10,9)$ & 74 & 19,1 & $(15,1-23,0)$ \\
\hline
\end{tabular}

En el grupo de 6-7 años no se registró la maloclusión; sólo 3 eran portadores de tratamiento de ortodoncia. En el interior de la tabla se dan porcentajes que suman siempre $100 \%$.

\section{Salud dental según sexo, residencia y nivel socio-económico}

En los tres grupos de edad y en ambas denticiones, los índices de caries (total y activa) son mayores entre los de NSE bajo
( $\mathrm{p}<0,05)$. Además, a los 14 años, las chicas presentan un mayor CAOD $(\mathrm{p}<0,05)$ por tener más obturaciones que los chicos $(\mathrm{p}<0,05)$ aunque tienen una tasa igual de caries activa (Tablas 9, 10 y 11).

Tabla 9. Prevalencia de caries, media y sus componentes (índice cod) en dentición temporal (6-7 años) (IC-95\%).

\begin{tabular}{|c|c|c|c|c|c|c|c|c|}
\hline \multirow[b]{2}{*}{ Total } & \multicolumn{2}{|r|}{$\% c$} & \multicolumn{2}{|r|}{$\%$} & \multicolumn{2}{|c|}{$\% \operatorname{cod}$} & \multicolumn{2}{|c|}{$\% \operatorname{cod}_{i}$} \\
\hline & 28,0 & $(24,2-31,9)$ & 17,5 & $(14,2-20,7)$ & 35,9 & $(31,8-40,0)$ & 41,3 & $(37,0-45,5)$ \\
\hline Hombre & 31,2 & $(25,6-36,8)$ & 15,2 & $(10,9-19,5)$ & 36,4 & $(30,6-42,2)$ & 39,4 & $(33,5-45,2)$ \\
\hline Mujer & 24,6 & $(19,2-29,9)$ & 19,8 & $(14,8-24,7)$ & 35,3 & $(29,3-41,2)$ & 43,2 & $(37,0-49,4)$ \\
\hline Urbano & 28,5 & $(24,0-33,1)$ & 16,8 & $(13,1-20,6)$ & 36,1 & $(31,2-40,9)$ & 41,0 & $(36,1-45,9)$ \\
\hline Rural & 26,4 & $(18,9-33,9)$ & 19,1 & $(12,4-25,8)$ & 35,2 & $(27,1-43,4)$ & 41,9 & $(33,5-50,3)$ \\
\hline Alto & $23,3^{*}$ & $(15,8-30,9)$ & 16,9 & $(10,2-23,6)$ & $31,4^{*}$ & $(23,1-39,7)$ & $38,7^{*}$ & $(30,0-47,4)$ \\
\hline Medio & 18,4 & $(11,4-25,5)$ & 16,8 & $(9,9-23,6)$ & 28,5 & $(20,3-36,8)$ & 31,0 & $(22,6-39,5)$ \\
\hline Bajo & 33,5 & $(27,8-39,3)$ & 18,1 & $(13,4-22,7)$ & 40,7 & $(34,8-46,7)$ & 46,4 & $(40,3-52,4)$ \\
\hline Media & & c & & $\mathbf{0}$ & & $\operatorname{cod}$ & & $\operatorname{cod}_{i}$ \\
\hline Total & 0,93 & $(0,75-1,11)$ & 0,33 & $(0,25-0,40)$ & 1,26 & $(1,06-1,46)$ & 1,51 & $(1,30-1,73)$ \\
\hline Hombre & 0,98 & $(0,73-1,22)$ & 0,33 & $(0,21-0,44)$ & 1,31 & $(1,03-1,59)$ & 1,53 & $(1,23-1,84)$ \\
\hline Mujer & 0,87 & $(0,61-1,13)$ & 0,32 & $(0,23-0,41)$ & 1,20 & $(0,91-1,48)$ & 1,48 & $(1,17-1,78)$ \\
\hline Urbano & 0,98 & $(0,77-1,20)$ & 0,30 & $(0,22-0,38)$ & 1,29 & $(1,05-1,53)$ & 1,55 & $(1,29-1,81)$ \\
\hline Rural & 0,76 & $(0,46-1,06)$ & 0,39 & $(0,22-0,56)$ & 1,16 & $(0,80-1,52)$ & 1,38 & $(1,00-1,75)$ \\
\hline Alto & $0,61^{*}$ & $(0,34-0,88)$ & 0,37 & $(0,20-0,53)$ & $0,98^{*}$ & $(0,62-1,34)$ & $1,20^{*}$ & $(0,82-1,59)$ \\
\hline Medio & 0,36 & $(0,18-0,55)$ & 0,36 & $(0,17-0,56)$ & 0,73 & $(0,46-1,01)$ & 0,87 & $(0,56-1,18)$ \\
\hline Bajo & 1,32 & $(1,01-1,62)$ & 0,29 & $(0,20-0,39)$ & 1,62 & $(1,29-1,94)$ & 1,92 & $(1,57-2,27)$ \\
\hline
\end{tabular}

i: incluye caries incipiente o supuestamente remineralizada

*: $\mathrm{p}<0,05$ 
Tabla 10. Prevalencia de caries, media y sus componentes (índice CAOD) en dentición permanente (11-12 años) (IC-95\%).

\begin{tabular}{|c|c|c|c|c|c|c|c|c|c|c|}
\hline & \multicolumn{2}{|r|}{$\% \mathrm{C}$} & \multicolumn{2}{|r|}{$\% A$} & \multicolumn{2}{|r|}{$\% 0$} & \multicolumn{2}{|c|}{ \%CAOD } & \multicolumn{2}{|c|}{$\% \mathrm{CAOD}_{\mathrm{i}}$} \\
\hline Total & 11,7 & $(8,8-14,5)$ & 0,8 & $(0,0-1,6)$ & 21,5 & $(17,9-25,2)$ & 28,5 & $(24,5-32,5)$ & 36,9 & $(32,6-41,2)$ \\
\hline Hombre & 10,4 & $(6,5-14,2)$ & 0,8 & $(0,0-1,9)$ & 22,8 & $(17,5-28,0)$ & 29,2 & $(23,5-34,8)$ & 36,4 & $(30,3-42,4)$ \\
\hline Mujer & 13,0 & $(8,7-17,3)$ & 0,8 & $(0,0-2,0)$ & 20,1 & $(15,0-25,3)$ & 27,7 & $(22,0-33,4)$ & 37,3 & $(31,2-43,5)$ \\
\hline Urbano & 11,2 & $(7,8-14,5)$ & 1,1 & $(0,0-2,2)$ & 21,6 & $(17,2-25,9)$ & 28,8 & $(24,0-33,6)$ & 37,1 & $(32,0-42,2)$ \\
\hline Rural & 12,7 & $(7,1-18,3)$ & 0,0 & $(0,0-0,0)$ & 21,2 & $(14,4-28,1)$ & 27,6 & $(20,1-35,1)$ & 36,1 & $(28,1-44,1)$ \\
\hline Alto & $7,8^{*}$ & $(3,3-12,2)$ & 0,7 & $(0,0-2,1)$ & 21,9 & $(15,0-28,9)$ & $26,9^{*}$ & $(19,5-34,3)$ & 36,8 & $(28,8-44,9)$ \\
\hline Medio & 9,4 & $(4,4-14,3)$ & 0,7 & $(0,0-2,1)$ & 15,2 & $(9,1-21,2)$ & 19,5 & $(12,8-26,2)$ & 27,5 & $(19,9-35,0)$ \\
\hline Bajo & 15,9 & $(10,9-20,9)$ & 0,9 & $(0,0-2,3)$ & 25,1 & $(19,1-31,0)$ & 35,2 & $(28,7-41,8)$ & 42,9 & $(36,1-49,7)$ \\
\hline Media & & C & & A & & $\mathbf{O}$ & & CAOD & & $\mathrm{CAOD}_{\mathrm{i}}$ \\
\hline Total & 0,19 & $(0,13-0,24)$ & 0,01 & $(0,0-0,02)$ & 0,43 & $(0,34-0,52)$ & 0,63 & $(0,52-0,73)$ & 0,89 & $(0,76-1,02)$ \\
\hline Hombre & 0,17 & $(0,09-0,25)$ & 0,00 & $(0,00-0,01)$ & 0,48 & $(0,34-0,61)$ & 0,66 & $(0,50-0,81)$ & 0,90 & $(0,71-1,08)$ \\
\hline Mujer & 0,20 & $(0,11-0,28)$ & 0,00 & $(0,00-0,02)$ & 0,37 & $(0,26-0,49)$ & 0,58 & $(0,43-0,73)$ & 0,87 & $(0,68-1,05)$ \\
\hline Urbano & 0,17 & $(0,11-0,23)$ & 0,01 & $(0,00-0,02)$ & 0,42 & $(0,32-0,52)$ & 0,60 & $(0,48-0,73)$ & 0,85 & $(0,70-1,00)$ \\
\hline Rural & 0,21 & $(0,08-0,35)$ & 0,00 & $(0,00-0,00)$ & 0,44 & $(0,27-0,61)$ & 0,66 & $(0,44-0,88)$ & 0,97 & $(0,70-1,23)$ \\
\hline Alto & $0,11^{*}$ & $(0,02-0,19)$ & 0,00 & $(0,00-0,02)$ & 0,45 & $(0,28-0,62)$ & $0,57^{*}$ & $(0,37-0,77)$ & $0,83^{*}$ & $(0,59-1,08)$ \\
\hline Medio & 0,10 & $(0,04-0,15)$ & 0,00 & $(0,00-0,02)$ & 0,31 & $(0,16-0,45)$ & 0,42 & $(0,24-0,59)$ & 0,59 & $(0,39-0,79)$ \\
\hline Bajo & 0,29 & $(0,17-0,41)$ & 0,00 & $(0,00-0,02)$ & 0,48 & $(0,34-0,62)$ & 0,78 & $(0,60-0,96)$ & 1,11 & $(0,89-1,32)$ \\
\hline
\end{tabular}

$\mathrm{i}$ : incluye caries incipiente o supuestamente remineralizada

*: $\mathrm{p}<0,05$

Tabla 11. Prevalencia de caries, media y sus componentes (índice CAOD) en dentición permanente (13-14 años) (IC-95\%).

\begin{tabular}{|c|c|c|c|c|c|c|c|c|c|c|}
\hline & \multicolumn{2}{|r|}{$\% \mathrm{C}$} & \multicolumn{2}{|r|}{$\% \mathrm{~A}$} & \multicolumn{2}{|r|}{$\% 0$} & \multicolumn{2}{|c|}{ \%CAOD } & \multicolumn{2}{|c|}{$\% \mathrm{CAOD}_{\mathrm{i}}$} \\
\hline Total & 14,9 & $(11,4-18,5)$ & 1,0 & $(0,0-2,0)$ & 38,9 & $(34,0-43,8)$ & 46,4 & $(41,4-51,4$ & 55,2 & $(50,2-60,1)$ \\
\hline Hombre & 15,1 & $(10,1-20,1)$ & 0,5 & $(0,0-1,5)$ & $32,3^{*}$ & $(25,7-38,8)$ & 41,9 & $(34,9-48,8)$ & 51,0 & $(43,9-58,0)$ \\
\hline Mujer & 14,7 & $(9,6-19,8)$ & 1,5 & $(0,0-3,3)$ & 45,7 & $(38,6-52,9)$ & 51,0 & $(43,8-58,2)$ & 59,4 & $(52,4-66,5)$ \\
\hline Urbano & 15,3 & $(11,0-19,7)$ & 0,7 & $(0,0-1,7)$ & 39,7 & $(33,7-45,6)$ & 46,8 & $(40,7-52,8)$ & 46,8 & $(40,7-52,8)$ \\
\hline Rural & 14,0 & $(7,7-20,3)$ & 1,6 & $(0,0-3,9)$ & 37,1 & $(28,4-45,9)$ & 45,4 & $(36,4-54,4)$ & 45,4 & $(36,4-54,4)$ \\
\hline Alto & $9,5^{*}$ & $(4,1-15,0)$ & 0,0 & $(0,0-0,0)$ & 36,5 & $(27,5-45,4)$ & $40,8^{*}$ & $(31,7-49,9)$ & $51,3^{*}$ & $(42,0-60,5)$ \\
\hline Medio & 11,8 & $(5,9-17,7)$ & 1,6 & $(0,0-4,0)$ & 33,8 & $(25,2-42,5)$ & 40,6 & $(31,6-49,6)$ & 46,6 & $(37,4-55,7)$ \\
\hline Bajo & 21,7 & $(15,0-28,3)$ & 1,3 & $(0,0-3,1)$ & 45,3 & $(37,3-53,4)$ & 55,9 & $(47,9-63,9)$ & 65,1 & $(57,4-72,7)$ \\
\hline Media & & $\mathrm{C}$ & & A & & 0 & & CAOD & & $\mathrm{CAOD}_{\mathrm{i}}$ \\
\hline Total & 0,31 & $(0,21-0,42)$ & 0,02 & $(0,0-0,03)$ & 0,95 & $(0,78-1,11)$ & 1,28 & $(1,06-1,50)$ & 1,82 & $(1,54-2,10)$ \\
\hline Hombre & 0,29 & $(0,16-0,42)$ & 0,01 & $(0,00-0,03)$ & $0,65^{*}$ & $(0,49-0,81)$ & $0,96^{*}$ & $(0,72-1,19)$ & $1,38^{*}$ & $(1,08-1,68)$ \\
\hline Mujer & 0,33 & $(0,16-0,50)$ & 0,02 & $(0,00-0,04)$ & 1,24 & $(0,96-1,53)$ & 1,60 & $(1,22-1,97)$ & 2,27 & $(1,79-2,75)$ \\
\hline Urbano & 0,28 & $(0,18-0,37)$ & 0,00 & $(0,00-0,01)$ & 0,95 & $(0,75-1,14)$ & 1,23 & $(1,01-1,46)$ & 1,67 & $(1,39-1,95)$ \\
\hline Rural & 0,38 & $(0,11-0,65)$ & 0,03 & $(0,00-0,07)$ & 0,93 & $(0,61-1,25)$ & 1,35 & $(0,84-1,86)$ & 2,13 & $(1,46-2,79)$ \\
\hline Alto & $0,20^{*}$ & $(0,00-0,42)$ & 0,00 & $(0,00-0,00)$ & 0,92 & $(0,60-1,23)$ & $1,13^{*}$ & $(0,67-1,58)$ & $1,46^{*}$ & $(0,97-1,96)$ \\
\hline Medio & 0,22 & $(0,09-0,34)$ & 0,01 & $(0,00-0,04)$ & 0,69 & $(0,47-0,91)$ & 0,93 & $(0,65-1,20)$ & 1,28 & $(0,92-1,64)$ \\
\hline Bajo & 0,47 & $(0,28-0,66)$ & 0,02 & $(0,00-0,06)$ & 1,17 & $(0,87-1,47)$ & 1,67 & $(1,28-2,06)$ & 2,50 & $(1,97-3,04)$ \\
\hline
\end{tabular}


Por lo que respecta al índice de restauración, no hay diferencias significativas ni por sexo ni por medio de residencia urbano/rural, pero sí las hay por nivel socio- económico: los de nivel bajo dan menores índices $(\mathrm{p}>0,05)$ a los 6 y 12 años, que se igualan a los 14 (Tabla 12).

Tabla 12. Índice de restauración (IR), por edades (IC-95\%).

\begin{tabular}{lllllll}
\hline \multicolumn{1}{c}{ IR } & \multicolumn{2}{c}{$\mathbf{6 - 7}$ (DT) } & \multicolumn{2}{c}{$\mathbf{1 1 - 1 2}$ (DP) } & \multicolumn{2}{c}{$\mathbf{1 3 - 1 4}$ (DP) } \\
\hline Total & 26,1 & $(20,6-31,6)$ & 68,9 & $(61,5-76,4)$ & 74,1 & $(67,6-80,6)$ \\
\hline Hombre & 25,2 & $(17,4-33,0)$ & 72,7 & $(62,3-82,9)$ & 68,1 & $(57,7-78,5)$ \\
Mujer & 27,1 & $(19,7-34,5)$ & 64,3 & $(53,3-75,3)$ & 78,0 & $(70,0-86,0)$ \\
\hline Urbano & 23,4 & $(12,4-34,4)$ & 69,7 & $(61,3-78,1)$ & 76,7 & $(70,2-83,2)$ \\
Rural & 34,2 & $(16,4-52,0)$ & 67,0 & $(52,1-81,9)$ & 68,9 & $(55,6-82,2)$ \\
\hline Alto & $37,7^{* 1}$ & $(25,4-50,0)$ & $79,0^{* 2}$ & $(66,1-91,9)$ & 81,5 & $(67,8-95,2)$ \\
Medio & 50,0 & $(33,1-66,9)$ & 74,1 & $(63,3-84,9)$ & 74,5 & $(63,1-85,9)$ \\
Bajo & 18,4 & $(12,9-23,9)$ & 61,3 & $(49,9-72,7)$ & 70,2 & $(60,8-79,6)$ \\
\hline
\end{tabular}

DT: dentición temporal

DP: dentición permanente

*: $\mathrm{p}<0,05$.

1: Comparación significativa: alto vs medio y medio vs bajo.

2: Comparación significativa: alto vs bajo.

No aparecen diferencias significativas en el estado periodontal, presencia de selladores y maloclusión (datos no mostrados).

\section{DISCUSIÓN}

El tipo de muestreo utilizado ha sido probabilístico (polietápico) y equiprobabilístico. Dado el carácter de obligatoriedad de la escolarización en Navarra, los escolares son la totalidad de la población diana. La negativa de un colegio a participar no debe considerarse que sesgue el estudio, pues ha sido por motivo administrativo. Respecto a la tasa de aceptación para ser explorados, no se produjo ninguna negativa por parte de los responsables de los escolares para participar en el estudio.

\section{El diagnóstico de caries en encuestas epidemiológicas}

La prevalencia de caries en los países occidentales industrializados ha experimentado un notable descenso en las últimas décadas ${ }^{12}$ y un reflejo de ello es la evolución experimentada tanto en Espa$\tilde{n} \mathrm{a}^{13}$ como en Navarra ${ }^{4}$ desde que disponemos de datos. La mayoría de los autores coinciden en que las razones pueden ser varias y complejas pero, entre ellas, quizás la más importante sea el uso extensivo de dentífricos fluorados ${ }^{12,14}$. Sin embargo, parte de este descenso también puede ser atribuido a cambios en el criterio diagnóstico $^{15}$.

El criterio diagnóstico es crítico en encuestas epidemiológicas de caries. Por esta razón, ya desde la $2^{\text {a }}$ edición (1997), se optó por aplicar dos niveles de diagnóstico de caries. Uno estandarizado, basado en los criterios de la OMS y otro ampliado, basado en principios clínicos, e incluía las lesiones incipientes y supuestamente remineralizadas que también son enfermedad de caries y que, dado el descenso en la prevalencia de esta enfermedad, empezaban a tomar una mayor relevancia. Este criterio ha sido refrendado por otros autores ${ }^{15}$. De hecho, varios han sido los intentos en la última década para desarrollar un sistema de medición de la caries dental que tuviera en cuenta todos los estadios clínicamente visuales y su 
estado de actividad ${ }^{16-18}$ y no sólo el estadio de caries claramente en dentina ${ }^{19}$.

La diferencia entre uno y otro es básicamente la necesidad de tratamiento operativo-restaurador inmediato: sí para el primero, no para el segundo. El criterio estandarizado de la OMS permite la comparación de resultados con otras encuestas tanto nacionales como internacionales y es un criterio imprescindible. El segundo, permitía ya desde su inicio hacer una proyección de futuro y revelar de un modo más ajustado a la realidad clínica de los individuos (pacientes), las necesidades de tratamiento tanto preventivas como operatorias. De hecho, este segundo nivel diagnóstico va a posibilitar la comparación con las nuevas metodologías de diagnóstico de caries en un futuro inmediato -que ya es presentecomo es el caso del Internacional Caries Detection \& Assessment System (ICDAS II) ${ }^{20}$. Diversos trabajos han sido publicados ya respecto de la validez y fiabilidad del siste$\mathrm{ma}^{21,22}$. El sistema ICDAS II, para aplicación tanto clínica como epidemiológica, permite a través de su sistematización convertir los diversos niveles de caries en opciones de tratamiento.

Así pues, en el informe se darán ambas cifras que vendrán separadas por una barra (criterio OMS / criterio ampliado; por ejemplo: CAOD / $\mathrm{CAOD}_{\mathrm{i}}$ ).

\section{Caries dental en 2007}

La prevalencia de caries en dentición temporal puede considerarse entre moderada y alta en términos de porcentaje de población afectada $(35,9 \% / 41,3 \%)$, pero baja en media de dientes afectados $(1,26 / 1,51)$, teniendo en cuenta que la dentición temporal la componen 20 dientes. Como ya es conocido en la distribución poblacional de esta enfermedad, un pequeño porcentaje de individuos acumula una mayoría de las lesiones; en este caso, el 14,7\% de los niños de 6 años acumula el 70,1\% de la lesiones de caries.

En dentición permanente, la prevalencia puede considerarse muy baja o baja a los 12 años $(28,5 \% / 36,9 \%)$, pero da un salto importante a los 14 años (46,4\%/55,2\%). Así también, la media de dientes afectados es muy baja a los $12(0,63 / 0,89)$ y llama la atención el incremento tan notable que se produce a los $14(1,28 / 1,82)$, donde la media de dientes afectados es el doble que tan sólo con dos años de edad menos.

Aquí también se repite el fenómeno de la distribución asimétrica poblacional, con un $16,4 \%$ de escolares acumulando el $80,6 \%$ de las lesiones (12 años); esto se muestra de otro modo en el $\mathrm{SiC}$ que es de 2,19 y 2,75 a los 12 y 14 años respectivamente. Dicho de otro modo, aun a pesar del descenso de la prevalencia de caries -ver epígrafe más adelante "Evolución en el período 19872007"-, sigue habiendo un porcentaje de población en donde este descenso no tiene un significado clínico apreciable. Son sujetos que siguen padeciendo mucho de caries; mientras que la media poblacional a los 12 años es 0,63, el SiC es 2,19 es decir, tres veces y media más.

Otro fenómeno importante que ha puesto de manifiesto este estudio -como ya adelantó el de 2002- es la gran diferencia existente entre la población originaria de España y los escolares de origen extranjero, diferencia que, como también se verá más adelante, es común en todo el país. Esta diferencia es particularmente importante en la dentición temporal (cod 0,9 vs 3,$47 ; \mathrm{p}<0,05$ ) que representa una media de dientes afectados 3,8 veces mayor, y se mantiene, aunque en menor grado, en dentición permanente a los 14 años (CAOD 1,16 vs 2,$28 ; \mathrm{p}<0,05)$ que representa una media casi 2 veces mayor. A este respecto, no parece que sea la condición de extranjero la determinante sino el NSE ya que el $75,5 \%$ de éstos están clasificados como NSE bajo.

Finalmente, un aspecto relevante es el de la distribución de la caries según el tipo de diente y según la superficie del diente afectada (mesial, oclusal, distal, bucal y lingual). En estas edades, y por lo que respecta a la dentición permanente, la caries afecta principalmente a los molares (82,5\% a los 12 y $64,1 \%$ a los 14 años) y, por superficie, la mayoría son caries oclusales (65\% aprox.). Esto es importante ya que indica, en el caso de 
la dentición permanente, una baja complejidad de las necesidades de tratamiento de esta población. Sin embargo, a este respecto es necesario señalar que el estudio produce una cierta infra-estimación de estas necesidades debido a las limitaciones, por un lado metodológicas de una encuesta poblacional -estandarización de criterios y medidas- y por otro de diagnóstico en un trabajo de campo en donde no es posible contar con información radiológica (también porque supondría un complejo problema de estandarización de toma y lectura de resultados). Con todo, se puede afirmar que las necesidades de tratamiento son bajas y de baja complejidad, como corresponde a una situación epidemiológica de baja prevalencia de caries. Caso distinto es el de la dentición temporal a los 6 años, en donde las necesidades y, sobre todo la complejidad, son algo mayores; en efecto, aquí las caries predominantes son las interproximales $(51,8 \%)$.

\section{Influencia del sexo, medio y nivel socio-económico}

Los dos elementos que cabe destacar son:

- El nivel de caries se sigue viendo afectado por el NSE.

- Desaparecen por completo las diferencias que había anteriormente según medio de residencia urbano/rural.

Respecto de lo primero, los escolares de NSE bajo presentan mayores índices de caries, total y activa, y en todos los niveles, ya sea de edad, de tipo de dentición y nivel diagnóstico, diferencias que son estadísticamente significativas en todos los casos. Por ejemplo, a los 12 años el CAOD de nivel alto-medio versus bajo es $0,57-0,42$ vs 0,78 $(\mathrm{p}<0,05)$ y el componente de caries activa es $0,11-0,10$ vs $0,29(\mathrm{p}<0,05)$. El hecho positivo en relación a esto es que todos reciben tratamiento en parecida medida, como lo demuestra el que no haya diferencias significativas en el componente obturado (número de empastes) en ningún grupo de edad ni tipo de dentición.

El segundo hecho relevante y que cumple uno de los objetivos del PADI es que el vivir en medio urbano o rural no afecta al nivel de caries ni al nivel de tratamiento: en todos los grupos de edad, tanto en porcentaje de sujetos que reciben obturación como en media de dientes obturados, las cifras son similares entre los residentes urbanos y rurales. Esto indica que los servicios dentales están cumpliendo con el objetivo de eliminar desigualdades en el acceso al tratamiento por razones de residencia y NSE.

En cuanto al sexo, cabe destacar que únicamente aparecen diferencias a los 14 años, en donde el CAOD es significativamente mayor en las chicas que en los chicos debido a que las primeras presentan más obturaciones que los chicos $(1,26$ vs $0,66 ; \mathrm{p}<0,05)$ aunque tiene la misma cantidad de caries activa $(0,34$ vs 0,30$)$.

\section{Evolución en el período 1987-2007}

Después del llamativo descenso de caries ocurrido en la década de los 90 , asistimos a un período de ralentización en el descenso, y estabilización según edades. La caries está estabilizada en dentición temporal a los 6 años desde 1997, tanto en porcentaje de población afectada como en media de dientes afectados (Tabla 13). Este fenómeno es común a otros países industrializados de occidente ${ }^{12}$. Sin embargo, en dentición permanente, continúa con una tendencia descendente, más moderada en los últimos años, pero estadísticamente significativa en el período 1997-2007, en los escolares de 12 años (CAOD 0,90 vs 0,63 ; $\mathrm{p}<0,05)$. En los de 14 años, este descenso es muy leve, no significativo y puede considerarse una estabilización.

Ahora bien, si descontamos el efecto de la altísima prevalencia de caries de la población de origen extranjero en los índices generales (ver más arriba), el descenso de caries es todavía mayor y puede apreciarse cómo es significativo en dentición temporal en el período $1997-2007$ (cod 1,16 vs 0,9; $\mathrm{p}<0,05)$. Este fenómeno se puso de manifiesto ya en la encuesta Navarra de 2002 y ha podido comprobarse en otras encuestas a nivel nacional ${ }^{4,23,24}$. 
Por último, un nuevo problema ha venido a añadirse a la dificultad de mostrar la historia de caries y, por tanto, su evolución a través del índice CAO, y es la presencia generalizada de obturaciones "blancas" de composite, de color muy parecido, cuando no igual, al del diente, que son difíciles de identificar. Este hecho podría estar produciendo una infra-estimación del componente O del $\mathrm{CAOD}^{12} \mathrm{y}$, por ende, del mismo CAOD.

\section{Tratamiento de la caries: índice de restauración}

Este índice experimentó un fuerte incremento en la década 1987-1997 tanto en dentición temporal como en permanente, sin duda por efecto del $\mathrm{PADI}^{5}$, hasta situarse en torno al $80 \%$ (aprox.), cifra que puede considerarse muy alta. Sin embargo, en la última década, ha ido descendiendo hasta situarse en torno al 70\% (aprox.). Será necesario estudiar el porqué de este descenso a pesar de que se produce en un contexto de gran asistencia al PADI y aumento de la red de profesionales concertados ${ }^{6}$. La primera apreciación es que el IR global está muy condicionado por los bajos niveles de tratamiento de la población de origen extranjero en 6 y 12 años con diferencias significativas $(\mathrm{p}<0,05)$, aunque se iguala en los de 14 años (Tabla 13).

Tabla 13. Evolución de la prevalencia de caries, media (cod/CAOD) e índice de restauración (IR) en el período 1987-2007.

\begin{tabular}{ccccccccccc}
\hline & \multicolumn{3}{c}{ Prevalencia } & \multicolumn{3}{c}{ cod / CAOD } & \multicolumn{3}{c}{ IR } \\
\hline & $6-7$ (DT) & $11-12$ & $13-14$ & $6-7$ (DT) & $11-12$ & $13-14$ & $6-7$ (DT) & $11-12$ & $13-14$ \\
1987 & $62,8^{*}$ & $68,8^{*}$ & $84,3 \mathrm{a}$ & $2,59^{*} \mathrm{a}$ & $2,30^{*} \mathrm{a}$ & $3,73 \mathrm{a}$ & $12,4^{*}$ & $42,2^{*}$ & 38,9 \\
1997 & 35,6 & 42,2 & $49,2 \mathrm{~b}$ & $1,16 \mathrm{~b}$ & $0,90 \mathrm{~b}$ & $1,35 \mathrm{~b}$ & 31,9 & 81,1 & 76,3 \\
2007 & 35,9 & 28,5 & $46,4 \mathrm{~b}$ & $1,26 \mathrm{~b}$ & $0,63 \mathrm{c}$ & $1,28 \mathrm{~b}$ & 26,1 & 68,9 & 74,1 \\
$2007 \mathrm{es}$ & 32,4 & 26,7 & $43,8 \mathrm{~b}$ & $0,90 \mathrm{c}$ & $0,58 \mathrm{c}$ & $1,16 \mathrm{~b}$ & 34,2 & 73,8 & 73,6 \\
\hline
\end{tabular}

En dentición temporal, las cifras son muy bajas: a los 6 años (26,1\% global; $34,2 \%$ en origen España) y son similares a la media de España de 2005 (22,9\% global; $27,7 \%$ en origen España) ${ }^{15}$. No obstante, este fenómeno es común en otros países y es más llamativo que países como Inglaterra o Francia, en donde el sistema público cubre este tratamiento, las cifras son extremadamente bajas (Inglaterra y Gales 5-6 años: 13\%; Francia 4 años: $6,2 \%)^{25,26}$ (Tablas 14 y 15).

Tabla 14. Caries y tratamiento en Navarra, España y algunas CCAA, a los 12 años.

\begin{tabular}{lcccc}
\hline & & \% CAOD & CAOD & IR \\
\hline Andalucía & 2001 & 49,6 & 1,38 & $43 \%$ \\
Baleares & 2005 & 45,4 & 0,89 & $55 \%$ \\
Cataluña & 2006 & 45,8 & 0,73 & $53 \%$ \\
Castilla-La Mancha & 2004 & 40,8 & 1,09 & $49 \%$ \\
Extremadura & 2001 & 50 & 1,31 & $36 \%$ \\
Navarra & 2007 & 28,5 & 0,63 & $69 \%$ \\
País Vasco & 2008 & 26,2 & 0,52 & $73 \%$ \\
Valencia & 2004 & 42,5 & 1,07 & $33 \%$ \\
España & 2005 & 47,2 & 1,33 & $53 \%$ \\
\hline
\end{tabular}


Tabla 15. Caries y tratamiento en Navarra y algunos países de la Unión Europea.

\begin{tabular}{lccccccc}
\hline & Año & Edad & CAOD & $\begin{array}{c}\text { CAOD } \\
\text { Regional }\end{array}$ & SiC & IR & $\begin{array}{c}\text { IR } \\
\text { Regional }^{*}\end{array}$ \\
\hline Alemania & 2004 & 12,5 & 0,98 & $0,71-1,42$ & 2,72 & $75 \%$ & $61-83 \%$ \\
Francia & 2006 & 12 & 1,23 & & & $49 \%$ & \\
Italia & $2004 / 05$ & 12 & 1,09 & $0,92-1,50$ & 2,99 & $33 \%$ & $23-44 \%$ \\
Reino Unido & $2004 / 05$ & 11,3 & 0,72 & $0,49-1,29$ & & $42 \%$ & $22-69 \%$ \\
Navarra & 2007 & 11,4 & 0,63 & - & 1,87 & $69 \%$ & - \\
\hline
\end{tabular}

* Valores máximo y mínimo de las diferentes regiones.

\section{Navarra en el contexto nacional e internacional de caries y tratamiento}

Se comparan las cifras de España y las diferentes comunidades autónomas que disponen de datos a partir del año 2000 (datos extraídos del Banco de Datos Epidemiológicos de $\mathrm{SESPO}^{27}$ ). País Vasco ${ }^{28}$ y Navarra son las comunidades que tienen un índice de caries más bajo a la vez que un nivel de tratamiento más alto (IR). El índice CAOD a los 12 años para España fue de 1,33 en 2005 , con un $53 \%$ de índice de restauración ${ }^{15}$. El nivel de caries en Navarra es netamente inferior $(0,63)$ mientras que el de tratamiento (69\%) es claramente superior a la media nacional y a casi todas las CC.AA. y es reflejo de la cobertura de este tratamiento por parte del Servicio Navarro de Salud/Osasunbidea (Tabla 14).

Tras casi veinte años de política sanitaria en salud oral en País Vasco y Navarra ${ }^{29}$ que ha combinado aspectos comunes como la aplicación de un mismo modelo asistencial y aspectos diferenciados como la política de fluoración ${ }^{30}$, parece haber llegado el momento de realizar una evaluación comparativa entre ambas comunidades.

En el contexto europeo, estos niveles se asemejan, e incluso están por encima, a los de las regiones mejor situadas de los países más avanzados de la Unión Europea, y son claramente superiores a la media de algunos, como Reino Unido ${ }^{31}$ Alemania $^{32}$, Francia $^{26}$ o Italia ${ }^{33}$. Llama la atención que países como Francia o Reino Unido, que tienen cobertura pública de estas presta- ciones, no alcancen a tratar ni siquiera el $50 \%$ de la caries (Tabla 15).

Las cifras de salud periodontal son pobres e indican, de forma indirecta, un bajo nivel de higiene dental. Sólo 2 de cada 10 escolares tiene unas encías completamente sanas, sin sangrado ni cálculo acumulado entre los dientes. A la mayoría $(62,8 \%)$ le sangran al sondaje suave lo que indica un bajo nivel de eficacia del cepillado dental (que provoca un cierto nivel de gingivitis por acumulación de placa) y otro $16,8 \%$ tiene ya cálculo. La situación afecta de forma generalizada, ya que no hay diferencias por ninguna de las variables sociodemográficas, ni por origen. Respecto a la evolución, suponen una mejora respecto de 2002 , aunque la presencia de cálculo se mantiene estable.

El sellado de fosas y fisuras es un procedimiento preventivo específico de caries y ha sido una prestación especialmente recomendada en el programa PADI. A los 12 y 14 años, el 40\% (aprox.) presenta algún sellado, con una media de 1,3-1,2 sellados lo que representa cifras similares a las de $2002(1,1-1,2)$ y ligeramente superiores a la media nacional $(1-1,05)$.

La necesidad de tratamiento (o bien son portadores actualmente de tratamiento) afecta aproximadamente a $1 / 3$ de esta población, cifra similar a la de encuestas anteriores, no hay variación. No hay diferencias significativas por variables socio-demográficas, pero las chicas llevan más ortodoncia que los chicos al igual que los de NSE altomedio respecto de los de NSE bajo. 


\section{CONCLUSIONES}

Consideración previa: aunque la población es un todo, de cara a analizar la evolución de estos indicadores de salud en la actual situación de importantes cambios socio-demográficos, es necesario hacer la distinción entre la población de origen autóctono -más o menos estable- y la de origen extranjero, en muchos casos recién llegada, mayoritariamente de bajo NSE y cuyos datos -con significativamente más enfermedad y menos tratamiento- enmascaran la evolución general. Hecha esta distinción:

- La caries continúa con su ritmo descendente aunque mucho más moderado que en la década anterior. Los niveles de caries en la población de origen extranjero son significativamente mayores y esta encuesta no permite analizar su evolución.

- Los índices de restauración, representativos del tratamiento de caries, con ser altos, descienden ligeramente.

- El NSE sigue condicionando la afectación por caries, mayor entre las clases sociales más desfavorecidas. Sin embargo, desaparecen las diferencias por medio urbano o rural.

- El resto de variables mantienen una tendencia estable.

- Navarra se sitúa entre las primeras regiones de España y Europa en salud oral de niños y adolescentes.

- Por último, estas conclusiones son válidas para la población general. Sin embargo, como se ha señalado, la enfermedad de caries tiene una distribución asimétrica y es necesario reconocer que, a pesar de los avances, sigue habiendo una capa de población que padece altos niveles de enfermedad, tiene elevadas necesidades de tratamiento y pertenece en su mayoría a niveles socio-económicos bajos.

\section{Agradecimientos}

Nuestro agradecimiento a todos los escolares de la muestra seleccionada y a sus familias por su consentimiento y participación. Asimismo, a los Centros Escolares por la autorización a utilizar sus instalaciones y a la Dirección General de Educación por su colaboración.

\section{BIBLIOGRAFÍA}

1. Cortés Martinicorena FJ, Abad Vicente FJ. Estudio epidemiológico de la salud bucodental de la población escolar de Navarra de 9 y 14 años de edad (1987). Arch Odontoestom Prev Comunit 1989; 1: 49-57.

2. Cortés J, Doria A, Martínez I, Asenjo MA, Sainz De Murieta I, Cuenca E. Prevalencia de caries dental entre la población infantil de Navarra, 1997. Arch Odontoestom Prev Comunit 1998; 14: 640-646.

3. Cortés J, Doria A, Martínez I, Asenjo MA, Sainz De Murieta I, Cuenca E. La caries dental de los escolares navarros según su medio de residencia y su nivel socio-económico. Evolución en el período 1987-1997. An Sist Sanit Navar 1999; 22: 205-211.

4. Cortés Martinicorena FJ, Doria Bajo A, Asenjo Madoz, MA, Sainz de Murieta, I, Ramón Torrell JM, Cuenca SAla E. Prevalencia de caries y estado periodontal de los niños y adolescentes de Navarra (2002). RCOE 2003; 8 (4): 381-390.

5. Cortés FJ, Ramón JM, CuEnca E. Doce años de Programa de Asistencia Dental Infantil (PADI) en Navarra (1991-2002). Utilización e indicadores de salud. An Sist Sanit Navar 2003; 26 (3): 373-382.

6. Departamento de Salud del Gobierno de Navarra [página web en internet]. Pamplona: Memoria del Servicio Navarro de SaludOsasunbidea 2006. [Acceso abril de 2008]. Disponible en: http://www.cfnavarra.es SALUD/publicaciones/SNSO/Memoria2006/ pdfs2006/capitulo_02.pdf

7. DíAz de Rada IgúZquiza V. Técnicas de análisis de datos para investigadores sociales. Aplicaciones prácticas con SPSS para Windows. Universidad Pública de Navarra. Madrid: RAMA Ed., 1999, pp. 110-115.

8. Instituto Nacional de Estadística [página web en internet]. Madrid: Ministerio de Sanidad y Consumo. Encuesta Nacional de Salud 2003. [Acceso enero 2005]. Disponible en: http:/ www.ine.es/prodyser/pubweb/saludyhs03/ saludyhs03.htm

9. WHO. Oral Health Surveys. Basic Methods. 3th edition. World Health Organization, Geneva 1987. 
10. Brathall D. Introducing the Significant Caries Index together with a proposal for a new global oral health goal for 12 year olds. Int Dent J 2000; 50: 378-384.

11. Doménech Massons JM. Métodos estadísticos en ciencias de la salud. Síntesis del curso. Barcelona: Universitat Autónoma de Barcelona, 1992.

12. MARThaler TM. Changes in dental caries 19532003. Caries Res 2004; 38: 173-181.

13. Bravo-Pérez M, Casals-Peidró E, Cortés-MartiniCORENA FJ, LlODRA-CAlvo JC. Encuesta de salud oral en España 2005. RCOE 2006; 11 (4): 409456.

14. Bratthall D, Hänsell-Petersson G, Sundberg H. Reasons for the caries decline: What do the experts believe? Eur J Oral Sci 1996; 104: 416422.

15. Pitts NB, Fejerskov O, von de Fehr FR. Caries epidemiology, with special emphasis on diagnostic standards. En: Fejerskov O, Kidd EAM (ed): Dental Caries. The disease and its clinical management. Blackwell Munksgaard 2003 Oxford : 141-163.

16. Nyvad B, Machiulskiene V, Baelum V. Relaibility of a new caries diagnostic system differentiating between active and inactive caries lesions. Caries Res 1999; 33: 252-260.

17. Fyffe HE, Deery CH, Nugent, ZJ, Nuttall NM, PITTS NB. Effect of diagnostic threshold on the validity and reliability of epidemiological caries diagnosis using the Dundee Selectable Threshold Method for caries diagnosis (DSTM). Community Dent Oral Epidemiol 2000; 28: 42-51.

18. Ismail AI, Sohn W, Tellez M, Amaya A, Sen A, Hasson $\mathrm{H}$ et al. The International Caries Detection and Assessment System (ICDAS): an integrated system for measuring dental caries. Community Dent Oral Epidemiol 2007; 35: 170-178.

19. Pitts NB, Evans DJ, Pine C. British Association for the study of Community Dentistry (BASCD) diagnostic criteria for caries prevalence surveys-1996/1997. Community Dental Health 1997; 14 (Suppl 1): 6-9.

20. ICDAS Foundation [página web en internet]. Dundee: Dental Health Services Research Unit. University of Dundee. [Acceso abril 2008]. Disponible en: http://www.icdas.org

21. Jablonski-Momeni A, Stachniss V, Ricketts DN, Heinzel-Gutenbrunner M, Pieper K. Reproducibility and accuracy of the ICDAS II for Deteccion of Oclusal Caries in vitro. Caries Res 2008; 42: 79-87.
22. Schoaib L, Deery C, Nugent ZJ, Ricketts DNJ. Reproducibility of ICDAS II Criteria for Oclusal and Approximal Caries Detection in Primary Teeth. Caries Res 2007; 41: 268-334.

23. Bravo-Pérez M, Llodra-Calvo JC, Cortés-MartiNicorena FJ, CASALS-PEIDRó E. Encuesta de salud oral de Preescolares en España 2007. RCOE 2007; 12 (3): 143-168.

24. Almerich-Silla JM, Montiel-Company JM. Influence of immigration and other factors on caries in 12- and 15-yr-old children. Eur J Oral Sci 2007; 115: 378-383.

25. Pitts NB, Boyles ZJ, Nugent N, Thomas N, Pine CM. The dental caries experience of 5-yearold children in England and Wales. Surveys co-ordinated by the British Association for the Study of Community Dentistry in 2001/2002. Community Dental Health 2003; 20: $45-54$.

26. Union Française pour la Santé Bucco-dentaire (UFSBD). La santé dentaire en France. Enfants de 6 et 12 ans. Monographie. UFSBD, Paris, 2006.

27. Banco de datos epidemiológicos [base de datos en internet]. Sociedad Española de Epidemiología y Salud Pública Oral (SESPO). [Acceso abril 2008] Disponible en: http:// www.infomed.es/sespo\%2Dbd

28. Llodra Calvo JC (Universidad de Granada). Gobierno Vasco. Departamento de Sanidad. Dirección de Salud Pública. Tercer estudio epidemiológico de la salud bucodental de la CAPV. Población escolar infantil 2008. Dirección de Salud Pública (en prensa).

29. VIÑES JJ. Políticas sanitarias en salud dental. An Sist Sanit Navar 2003; 26: 341-343.

30. Freire JM. El Pograma Dental de Atención Infantil (PADI) de Navarra y del País Vasco: logros y nuevas metas. An Sist Sanit Navar 2003; 26: 423-428.

31. Pitts NB, Boyles ZJ, Nugent N, Thomas N, Pine $\mathrm{CM}$. The dental caries experience of 11-yearold children in Great Britain. Survey coordinated by the British Association for the study of Community Dentistry in 2004/2005. Community Dental Health 2006; 23: 44-57.

32. Schulte AG, Momeni A, Pieper K. Caries prevalence in 12-year-old children from Germany. Results of the 2004 national survey. Community Dental Health 2006; 23: 197-202.

33. Campus G, Solinas G, Cagetti MG, Senna A, MineLLI L, MAJORI S et al. National Pathfinder Survey of 12-year-old Children's Oral health in Italy. Caries Res 2007; 41: 512-517. 
